\title{
Psychiatrie und Glaube
}

Wie jeder Prophet des Alten Testaments hört auch Ezechiel die Stimme Gottes und versucht, diese von Gott empfangene Botschaft seinem Volk mitzuteilen. Ezechiel hört die Stimme Gottes häufiger als jeder andere Prophet, auch beschreibt er befehlende, kommentierende und dialogische Stimmen, die über ihn sprechen. In den gegenwärtig gültigen psychiatrischen Diagnosesystemen sind solche pathologischen Wahrnehmungen charakteristisch für schizophrene Störungen, sofern sie wenigstens ein Monat lang auftreten.

Wir behandeln immer wieder PatientInnen, die die Stimme des Teufels zu hören glauben, oder spüren, sich nächtlich mit Jesus Christus geschlechtlich zu vereinigen, oder überzeugt sind, vom Teufel besessen zu sein. Wenn diese Symptome häufig genug oder andauernd sind, behandeln wir diese Menschen als an Schizophrenie erkrankt. PsychiaterInnen und PsychotherapeutInnen gehen erfahrungsgemäß einfühlsam mit gläubigen PatientInnen der eigenen und anderer Religionen um. Sie lernen in ihrer Ausbildung die Wichtigkeit der Toleranz gegenüber Andersdenkenden. Bei gewissen religiösen Wahnthemen können aber auch heute und gerade bei tiefgläubigen Behandlern Abgrenzungsprobleme auftreten. Wir versuchen im Donauspital den Bereich des persönlichen Glaubens von der medizinischen Psychiatrie streng zu trennen. So ist dem ärztlichen Personal z. B. das gemeinsame Beten mit Patienten im Bereich der Abteilung nicht erlaubt. Es muss erwähnt werden, dass eine Diskussion zu diesem Aspekt 2011 im British Journal of Psychiatry (199: 94-98) durchaus kontroversiell diskutiert wurde.

Es ist der Fortschritt der Naturwissenschaften und der psychiatrischen und psychotherapeutischen Wissenschaften ganz allgemein, der die Interpretation und die Bedeutung auch alter religiöser Texte verändert hat. Immanuel Kant schreibt. „Aberglaube ist der Hang, in das was als nicht natürlicher Weise zugehend vermeint wird, ein größeres Vertrauen zu setzen, als was sich nach Naturgesetzen erklären lässt ..... Es ist also der Fortschritt der Naturwissenschaften und der psychiatrischen und psychotherapeutischen Wissenschaften ganz allgemein, der die Interpretation und die Bedeutung auch alter religiöser Texte verändert hat. Allerdings ist das Wissen um die biologischen und tiefenpsychologischen Grundlagen psychiatrischer Erkrankungen bei weitem nicht so weit fortgeschritten, wie das Wissen des Neurologen z. B. über die Epilepsie. Kein Neurologe glaubt angesichts des großen Wissens um die Pathophysiologie epileptischer Entladungen im Schläfenlappen noch, dass PatientInnen mit Epilepsie von bösen Geistern oder Dämonen besessen sein könnten. Naturwissenschaftliche Methoden, insbesondere aus dem Bereich der Bildgebung (z. B. fMRI), legen heute naturwissenschaftliche Erklärungen für das Phänomen der akustischen Halluzinationen nahe.

Die Gesellschaft und insbesondere kirchliche Kreise ohne psychiatrische Ausbildung sind weiterhin mehrheitlich überzeugt, dass es den Teufel und Hexen gibt, und dass es auch vom Teufel besessene Menschen gibt, denen nicht die Psychiatrie, sondern die Kirche oder der liebe Gott helfen solle. Auch Kardinal Schönborn betonte diese Sichtweise wiederholt in der Kolumne Sonntagsevangelium der Kronenzeitung. In diesem Spannungsfeld findet heute auch in der westlich zivilisierten Welt Psychiatrie und Psychotherapie statt. Zwei Artikel der vorliegenden Ausgabe der Psychopraxis nähern sich den interessanten Grenzbereichen von Glauben, Aberglauben, Wahn und Religion. Eine zum Nachdenken anregende Lektüre wünscht Ihnen

\section{Peter Fischer}

